\title{
DNA methylation changes in the postmortem dorsolateral prefrontal cortex of patients with schizophrenia
}

\author{
Shusuke Numata ${ }^{1,2}$, Tianzhang Ye ${ }^{3}$, Mary Herman ${ }^{1}$ and Barbara K. Lipska ${ }^{1 *}$ \\ 1 Human Brain Collection Core, National Institute of Mental Health, National Institutes of Health, Bethesda, MD, USA \\ 2 Department of Psychiatry, Course of Integrated Brain Sciences, Medical Informatics, Institute of Health Biosciences, The University of Tokushima, Tokushima, \\ Japan \\ ${ }^{3}$ The Lieber Institute for Brain Development, Johns Hopkins University Medical Center, Baltimore, MD, USA
}

\section{Edited by:}

Marta Barrachina, Bellvitge

Biomedical Research Institute, Spain

\section{Reviewed by:}

Alexandra M. Lopes, University of

Porto, Portugal

Ilan A. Kerman, University of

Alabama at Birmingham, USA

\section{*Correspondence:}

Barbara K. Lipska, Human Brain Collection Core, National Institute of Mental Health, 10 Center Drive, $R$. 4N306, Bethesda, MD 20892-1385, USA

e-mail: lipskab@mail.nih.gov
Background: Schizophrenia is a complex psychiatric disorder with a lifetime morbidity rate of $0.5-1.0 \%$. The pathophysiology of schizophrenia still remains obscure. Accumulating evidence indicates that DNA methylation, which is the addition of a methyl group to the cytosine in a $\mathrm{CpG}$ dinucleotide, might play an important role in the pathogenesis of schizophrenia.

Methods: To gain further insight into the molecular mechanisms underlying schizophrenia, a genome-wide DNA methylation profiling (27,578 CpG dinucleotides spanning 14,495 genes) of the human dorsolateral prefrontal cortex (DLPFC) was conducted in a large cohort $(n=216)$ of well characterized specimens from individuals with schizophrenia and non-psychiatric controls, combined with an analysis of genetic variance at $\sim 880,000$ SNPs.

Results: Aberrant DNA methylation in schizophrenia was identified at $107 \mathrm{CpG}$ sites at $5 \%$ Bonferroni correction $\left(p<1.99 \times 10^{-6}\right)$. Of these significantly altered sites, hyper-DNA methylation was observed at 79 sites (73.8\%), mostly in the $\mathrm{CpG}$ islands (CGIs) and in the regions flanking CGIs (CGI: 31 sites; CGI shore: 35 sites; CGI shelf: 3 sites). Furthermore, a large number of cis-methylation quantitative trait loci ( $\mathrm{mQTL}$ ) were identified, including associations with risk SNPs implicated in schizophrenia.

Conclusions: These results suggest that altered DNA methylation might be involved in the pathophysiology and/or treatment of schizophrenia, and that a combination of epigenetic and genetic approaches will be useful to understanding the molecular mechanism of this complex disorder.

Keywords: schizophrenia, DNA methylation, SNP, postmortem, array, expression

\section{INTRODUCTION}

Schizophrenia is a complex psychiatric disorder with a lifetime morbidity rate of $0.5-1.0 \%$. Accumulating evidence indicates that DNA methylation, which is the addition of a methyl group to the cytosine in a $\mathrm{CpG}$ dinucleotide, might play an important role in the pathogenesis of schizophrenia. For example, L-methionine, a precursor of S-adenosylmethionine, which donates its methyl group to various acceptors, exacerbates the psychotic symptoms of schizophrenia patients (Pollin et al., 1961; Cohen et al., 1974). L-methionine-treated mice exhibited increased DNA methylation that was accompanied by decreased mRNA levels of specific genes, and by behavioral changes similar to those seen in schizophrenia (Tremolizzo et al., 2002, 2005). In addition, an increased mRNA expression of DNA methyl-transferases (DNMT1 and DNMT3a) has been observed in schizophrenia (Veldic et al., 2004, 2005; Ruzicka et al., 2007; Zhubi et al., 2009). Furthermore, aberrant DNA methylation in brains of patients with schizophrenia (Abdolmaleky et al., 2005, 2006, 2011; Grayson et al., 2005; Iwamoto et al., 2005; Tamura et al., 2007; Mill et al., 2008;
Tolosa et al., 2010; Wockner et al., 2014) and the associations of different DNA methylation patterns with phenotypic discordance of schizophrenia between twins (Petronis et al., 2003; Dempster et al., 2011; Kinoshita et al., 2013) have been reported. However, the sample sizes in these previous epigenetic studies of schizophrenia were relatively small and the number of CpG sites interrogated was limited.

Tissue-specific differences in DNA methylation have been extensively documented (Christensen et al., 2009; Davies et al., 2012). Thus, since schizophrenia is a brain disorder, it is particularly important to examine the epigenetic modifications of the brains in patients with schizophrenia, rather than in the peripheral tissues. In this study, we conducted a genome-wide DNA methylation profiling (27,578 CpG dinucleotides spanning 14,495 genes) of the dorsolateral prefrontal cortex (DLPFC), a brain region implicated in cognition and schizophrenia, in a large cohort of well characterized specimens (106 patients with schizophrenia and 110 non-psychiatric controls), and identified genes whose methylation levels differed between patients with 
schizophrenia and controls. We also conducted a genome-wide association analysis of single nucleotide polymorphisms (SNPs) with DNA methylation in the same samples, and revealed a large number of cis-methylation quantitative trait loci (mQTL), including associations with risk SNPs implicated in schizophrenia. These results will add further insight into the molecular mechanisms of the pathophysiology of schizophrenia.

\section{MATERIALS AND METHODS HUMAN POSTMORTEM BRAIN TISSUE COLLECTION}

Postmortem human brains $(n=185)$ were collected through the Offices of the Chief Medical Examiners of Washington, DC and Virginia, Northern District by the Section on Neuropathology at the Clinical Brain Disorders Branch, National Institute of Mental Health, National Institutes of Health (NIH), according to the NIH Institutional Review Board (IRB) and ethical guidelines under protocol \#90-M-0142. Thirty one additional postmortem human brain specimens were collected through the Stanley Medical Research Institute. Clinical characterization, neuropathological screening, toxicological analyses, and dissections of the DLPFC were performed as previously described (Lipska et al., 2006). Briefly, all patients met DSM-IV criteria for a lifetime Axis I diagnosis of schizophrenia $(n=97)$ or schizoaffective disorder $(n=9)$ according to DSM-IV, and controls were defined as those individuals with no history of significant psychological problems or psychological care, psychiatric admissions, or drug detoxification and with no known history of psychiatric symptoms or substance abuse, as determined by both telephone screening and medical examiner documentation as well as negative toxicology results. Demographic data for these samples are summarized in Supplementary Table S1.

\section{GENOTYPING METHODS}

SNP genotyping with Human1M-Duo V3 BeadChips (Illumina Inc., San Diego, CA) was carried out according to the manufacturer's instructions, using DNA extracted from cerebellar tissue. Genotype data were analyzed using the Genotyping Analysis Module within the BeadStudio software (Illumina Inc.). For data analysis, 875,511 SNPs with missing calls $<2 \%$, HardyWeinberg equilibrium $p$-values $\geq 0.001$, and minor allele frequencies $\geq 0.015$ were used, from among a total of $1,199,187$ SNPs.

\section{METHYLATION METHODS}

Genomic DNA was extracted from $100 \mathrm{mg}$ of pulverized DLPFC tissue using the phenol-chloroform method. Bisulfite conversion of $600 \mathrm{ng}$ genomic DNA was performed using the EZ DNA methylation kit (Zymo Research). Methylation of DNA extracted from the DLPFC was assessed according to the manufacturer's instructions using Infinium HumanMethylation27 BeadChips (Illumina Inc.). Quantitative measurements of DNA methylation were determined for 27,578 CpG dinucleotides spanning 14,495 genes. CpG sites were selected by Illumina Inc. in the gene promoter regions, within $1 \mathrm{~kb}$ upstream and 500 bases downstream of the transcription start sites (TSSs). CGIs, CGI shores (0-2 kb from CGIs), CGI shelves (2-4 kb from CGIs) were defined as in a previous paper (Irizarry et al., 2009). DNA methylation data were analyzed using the Methylation Analysis Module within the BeadStudio software (Illumina Inc.). Normalization was carried out using lumiMethyN function from lumi package. The DNA methylation level of each $\mathrm{CpG}$ site was calculated as an $M$-value, which is the $\log 2$ ratio of methylated and unmethylated probe intensities. The technical schemes of this array have been described in detail in a previously published paper (Bibikova et al., 2009). Qualified CpG sites used in statistical analyses were defined as follows: (1) detected in $80 \%$ subjects, (2) excluded sex chromosome, (3) excluded $100 \%$ non-specific probes that completely match to other sequences, (4) excluded probes with SNPs at the CpG site with minor allele frequency MAF $>0.1 \%$. A list of potential non-specific probes and polymorphic probes of Illumina Human 27K Methylation Array can be downloaded at http://braincloud.jhmi.edu/downloads.htm. The final data set included 25,156 CpG sites (Supplementary Table S2). To ensure data reproducibility, 10 samples were analyzed in duplicates starting from the bisulfite conversion step, and high reproducibility was observed ( $r^{2}$ ranged from 0.9973 to 0.9921$)$. For validation, we used 92 samples from the current study and measured methylation status at $34 \mathrm{CpG}$ sites using Illumina custom GoldenGate platform. The CpG site positions for the probes were exactly the same as in the Infinium arrays. The correlation between the data from the two platforms was very high $\left(r^{2}=0.79\right)$ as described in Numata et al. (2012).

\section{STATISTICAL METHODS}

Surrogate variable analysis was used to account for known and unknown factors affecting the data, including batch effects (Leek and Storey, 2007). A general linear model was then used to examine the effects of the primary variables: age, sex, race, and diagnosis as well as the surrogate variables. The residuals from multiple regression analysis were used to analyze associations with SNP genotypes by PLINK (Purcell et al., 2007). Outliers were identified using Grubb's test and removed from further analysis. SNPs within $1 \mathrm{Mb}$ of a CpG site were defined as cis-SNPs, as in previous studies (Gibbs et al., 2010; Zhang et al., 2010; Numata et al., 2012). In these analyses, Bonferroni correction for multiple testing was applied at the 0.05 level.

\section{TRANSCRIPTION METHODS}

For correlations between DNA methylation and expression, we utilized previously published expression data from the DLPFC obtained using Human HT-12_V3 Illumina BeadArrays as described in detail in Ye et al. (2012). Only probes expressed above the background $(p<0.05)$ in at least $80 \%$ of subjects were analyzed. Normalization was carried out using lumi R package. The ComBat R package was used for batch effects. Surrogate variable analysis was used with SVA R package with age, sex, race, and diagnosis as primary variables. A step-wise model selection was used for each gene, and a multiple linear regression analysis was performed with the best fit model.

\section{RESULTS}

\section{DIAGNOSTIC DIFFERENCES IN DNA METHYLATION}

Significant diagnostic differences in DNA methylation were observed at $107 \mathrm{CpG}$ sites at $5 \%$ Bonferroni correction $(p<$ $1.99 \times 10^{-6}$, Supplementary Table S3) out of a total 25,156 CpG 


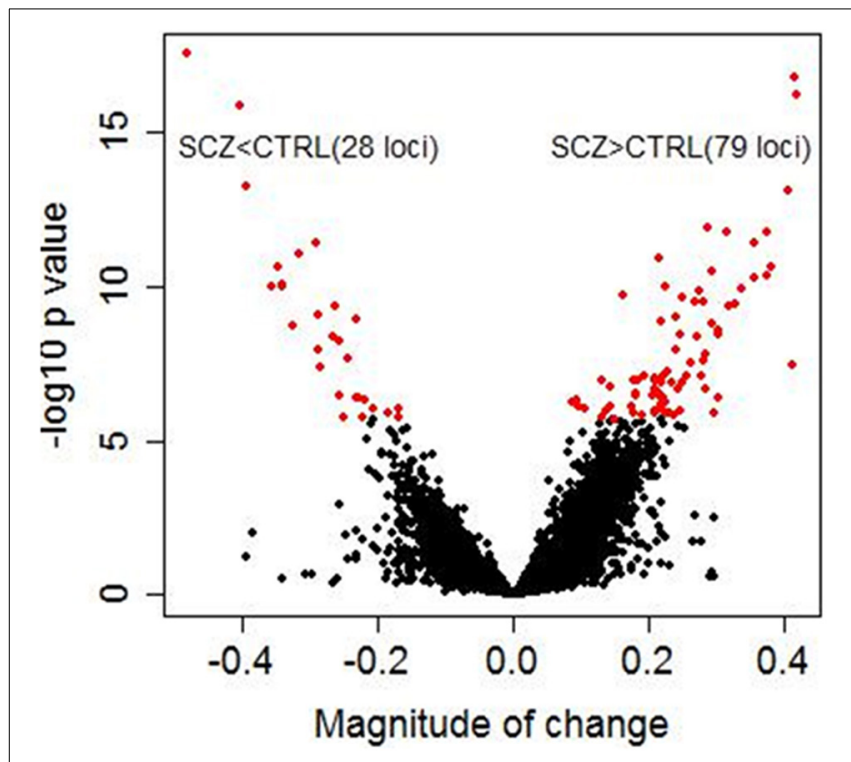

FIGURE 1 | Diagnostic differences in DNA methylation. The $x$ axis represents magnitude of a change (beta value) between controls and schizophrenia subjects calculated by linear regression analysis in 216 samples. The y axis represents $-\log 10 p$-values. Each dot represents an individual CpG site (a total of 25,156 CpG sites). Red dots represent 107 $\mathrm{CpG}$ sites that showed significant diagnostic differences between schizophrenic subjects and controls (5\% Bonferroni correction). Magnitude of change $>0$ corresponds to higher methylation in patients with schizophrenia than in controls, whereas magnitude of change $<0$ corresponds to lower methylation in patients with schizophrenia than in controls. Of these $107 \mathrm{CpG}$ sites, 79 sites (73.8\%) demonstrated higher methylation in patients with schizophrenia than in controls.

sites examined. Of these $107 \mathrm{CpG}$ sites, 79 sites (73.8\%) demonstrated higher DNA methylation in schizophrenia than in controls (Figure 1). Examples of diagnostic-biased genes include GRIA4 (glutamate receptor, inotropic, AMPA4) and ASTN2 (astrotactin 2) (Figure 2), both of these genes have been previously implicated in schizophrenia (Makino et al., 2003; Vrijenhoek et al., 2008; Wang et al., 2010). When the 79 sites hypermethylated in schizophrenia were classified into four categories (CGI, CGI shore, CGI shelf, and others), most of them (87.3\%) were located in the CGIs and in the regions flanking CGIs (CGI: 31 sites; CGI shore: 35 sites; CGI shelf: 3 sites; others: 9 sites). In contrast, when 28 sites, which demonstrated significantly reduced DNA methylation in schizophrenia, were classified into the same four categories, most of them $(85.7 \%)$ were located not in or near CGIs but outside CGIs (i.e., others: 23 sites; CGI: 1 site; CGI shore: 3 sites; CGI shelf: 1). There was no effect of smoking on DNA methylation in any group.

\section{THE mOTL ANALYSIS}

A genome-wide association analysis of SNPs with DNA methylation was conducted in the combined cohort of controls and cases to identify mQTL, as has been done in other previous QTL studies (Liu et al., 2010; Zhang et al., 2010; Gamazon et al., 2013). In the cis-analysis (cis defined as within $1 \mathrm{Mb}$ of a CpG site), 36,366 SNP-CpG pairs were significantly correlated (Bonferroni corrected $p<0.05$ ) (Supplementary Table S4). When the association analysis was performed in the subjects with schizophrenia and controls separately, $89.7 \%$ of cis-associations from the subjects with schizophrenia and $89.5 \%$ of cis-associations from controls were found in the analysis of the combined cohort (Supplementary Table S5).

Among these significant cis-associations in the combined cohort, we found SNPs that have been previously associated with increased risk for schizophrenia. For instance, as shown in the recent study (Smith et al., 2013), a frequent promoter variant rs6311 of the HTR2A [5-hydroxytryptamine (serotonin) receptor 2A] gene, widely implicated in human neuropsychiatric disorders and significant in the meta analysis in the Schizophrenia Gene database (Allen et al., 2008), decreases usage of an upstream transcription site encoding a longer $5^{\prime}$ UTR with greater translation efficiency. Our data show that the A allele, which increases risk for schizophrenia, is associated with higher methylation at the HTR2A locus cg00308665 (Bonferroni corrected $p=$ $\left.3.02 \times 10^{-16}\right)$. These data suggest a possible interplay between higher CpG methylation and repressed expression of the extended $5^{\prime}$ UTR and might offer a molecular mechanism for the statistical association of rs6311 with mental disorders.

Two other SNPs, rs13219354 (PRSS16) and rs3747600 (C16orf5), with significant mQTLs in our analysis, were found among the top 18 markers in another large genome-wide association study of schizophrenia (a total of 12,945 cases and 34,591 controls) by Stefansson et al. (2009). One SNP, rs7914558 (CNNM2), which was significant after Bonferroni correction in our analysis, appeared among the top 10 markers in the mega-analysis combining genome-wide association study data by the Schizophrenia Psychiatric Gnome-Wide Association Study Consortium (2011) (a total of 17,836 cases and 33,859 controls). This intronic SNP in CNNM2 (rs7914558) predicted DNA methylation status at the cg15439196 CpG site in CNNM2 and at cg00035347 site of the neighboring gene, NT5C2 (5' nucleotidase, cytosolic II), Bonferroni corrected $p=1.74 \times 10^{-2}$ and $6.05 \times 10^{-22}$, respectively), Figure 3 .

\section{DNA METHYLATION CORRELATES WITH EXPRESSION OF A SUBSET OF GENES}

To interrogate relationships between DNA methylation and transcription, we correlated (Pearson's correlation) methylation status at $\mathrm{CpG}$ loci in gene promoters with expression of all probes on the array corresponding to the same gene using genome-wide transcriptional profiling data of the DLPFC from the same subjects (Ye et al., 2012) (all pairs were within $100 \mathrm{~kb}$, a total number of such correlations was 107,031). Overall, across all CpG sites, there was poor correlation with expression. We identified only 86 correlations significant after Bonferroni correction $\left(p<4.63 \times 10^{-7}\right)$, of which $73(84.9 \%)$ were negative (Supplementary Table S6). Of particular interest is GNMT (glycine N-methyltransferase) with high negative correlation between DNA methylation and expression $\left(r=-0.42, p=4.5 \times 10^{-10}\right)$. GNMT catalyzes the synthesis of sarcosine from glycine using S-adenosylmethionine as the methyl-donor in one carbon metabolic pathway, and $\mathrm{Gnmt}^{-/-}$mice have shown dysregulation of DNA methyltransferases (DNMT1 and Dnmt3a), ErbB (Nrg1 and ErbB4), and 


\section{$\operatorname{cg} 19343464$}

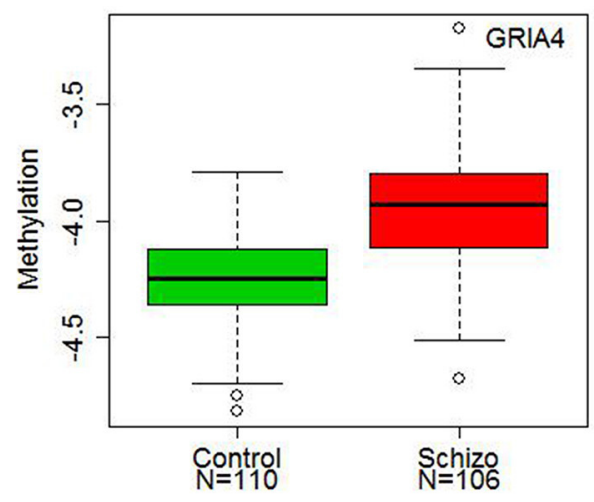

FIGURE 2 | Diagnostic differences in the DNA methylation levels of GRIA4 and ASTN2. The $x$ axis represents diagnostic groups (control and schizophrenia), and the $y$ axis represents DNA methylation levels (log2 ratio of methylated and unmethylated probe intensities). The lines in the bar graph indicate the median, and the bars (whiskers)

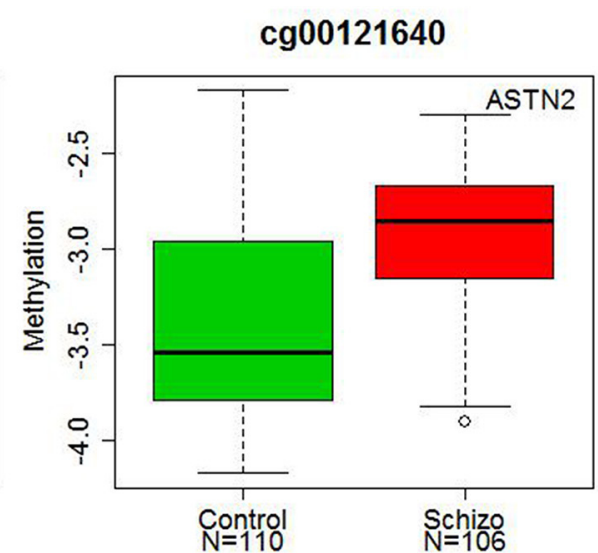

represent the minimum and maximum values after the removal of the outliers. The CpG sites of the GRIA4 (cg19343464) and ASTN2 (cg00121640) showed significantly higher DNA methylation in patients with schizophrenia than in controls $\left(p=3.55 \times 10^{-7}\right.$ and $\left.p=1.09 \times 10^{-6}\right)$.
mTOR (Akt2, S6, S6k1, and S6k2) in the cortex, as well as abnormal behaviors associated with schizophrenia (Yang et al., 2012).

\section{DISCUSSION}

We identified over one hundred CpG sites with aberrant DNA methylation in schizophrenia in this genome-wide DNA methylation profiling study performed in a large cohort of 106 patients with schizophrenia and 110 non-psychiatric controls. To date, 11 DNA methylation studies were conducted on schizophrenia using postmortem brains, but the sample sizes of these studies were relatively small ( $\sim 35$ patients with schizophrenia) (Abdolmaleky et al., 2005, 2006, 2011; Grayson et al., 2005; Iwamoto et al., 2005; Dempster et al., 2006; Tamura et al., 2007; Mill et al., 2008; Tochigi et al., 2008; Tolosa et al., 2010; Wockner et al., 2014).

Our study demonstrated that altered DNA methylation in schizophrenia was more likely to show a pattern of hyper- DNA methylation, and that it occurred at CpG sites not only in the CGIs but also in the CGI shores. These findings are in agreement with a recent genome-wide DNA methylation study using the leukocytes of patients with schizophrenia (Kinoshita et al., 2013). In line with our results, an increased mRNA expressions of DNA methyl-transferases has been found in post-mortem brains of schizophrenia (Veldic et al., 2004, 2005; Ruzicka et al., 2007; Zhubi et al., 2009). We identified a number of genes with significant epigenetic alterations in schizophrenia, and some of these genes, such as GRIA4, ASTN2, and DCDC2 (doublecortin domain containing 2) with increased DNA methylation at particular CpG loci, have previously been implicated in schizophrenia. For example, genetic variations in GRIA4, a subunit of AMPA receptor that mediates fast synaptic excitatory neurotransmission, have been associated with schizophrenia and antipsychotic responses in patients. Moreover, GRIA4-deficient mice exhibit schizophreniarelated phenotypes (Makino et al., 2003; Lavedan et al., 2009; Sagata et al., 2010; Fijal et al., 2012). ASTN2 is expressed at high levels in migrating cerebellar granule neurons at developmental stages when glial-guided migration occurs (Wilson et al., 2010). SNPs in this gene have been associated with schizophrenia and metabolic side effects of antipsychotic drugs, as well as with autism, attention deficit hyperactivity disorder, hippocampal volume, and cognition (Lesch et al., 2008; Vrijenhoek et al., 2008; Glessner et al., 2009; Wang et al., 2010; Adkins et al., 2011; Lionel et al., 2011; Bis et al., 2012). DCDC2 gene is located on chromosome 6p22.1, a region with strong evidence of linkage with schizophrenia (Shi et al., 2009). This gene has been also identified as a candidate gene for reading disability, and implicated in neuronal migration (Meng et al., 2005). SNPs in this gene have been associated with cortical gray matter and resting state fMRI activity in language-related brain regions in patients with schizophrenia (Jamadar et al., 2011, 2013).

We did not find changes in methylation status for a number of genes reported in the previous postmortem brain studies of DNA methylation based on candidate gene approaches. For instance hyper- DNA methylation of RELN, SOX10 [SRY (sex determining region Y)-box 10], FOXP2 (forkhead box P2), and HTR2A as well as hypo- DNA methylation of MB-COMT (membrane-bound catechol-O-methyltransferase) and HTR2A have been reported in schizophrenia (Grayson et al., 2005; Iwamoto et al., 2005; Abdolmaleky et al., 2006, 2011; Tolosa et al., 2010). Although Infinium HumanMethylation27 BeadChips covered these five genes, the exact locations of CpG sites were different from those in the previous studies. This may explain discrepancies between our results and those in the previous studies. When we compared our data with the previous genomewide DNA methylation study using CpG-island microarrays (Mill et al., 2008), we found one common gene, MRPS14 (mitochondrial ribosomal protein S14), which showed significantly higherDNA methylation changes in schizophrenia in both studies. This result suggests that there may be changes in mitochondrial function in schizophrenia (Anglin et al., 2012). Recently, Wockner et al. identified 4641 probes corresponding to 2929 genes which were found to be differentially methylated between diagnosis in 


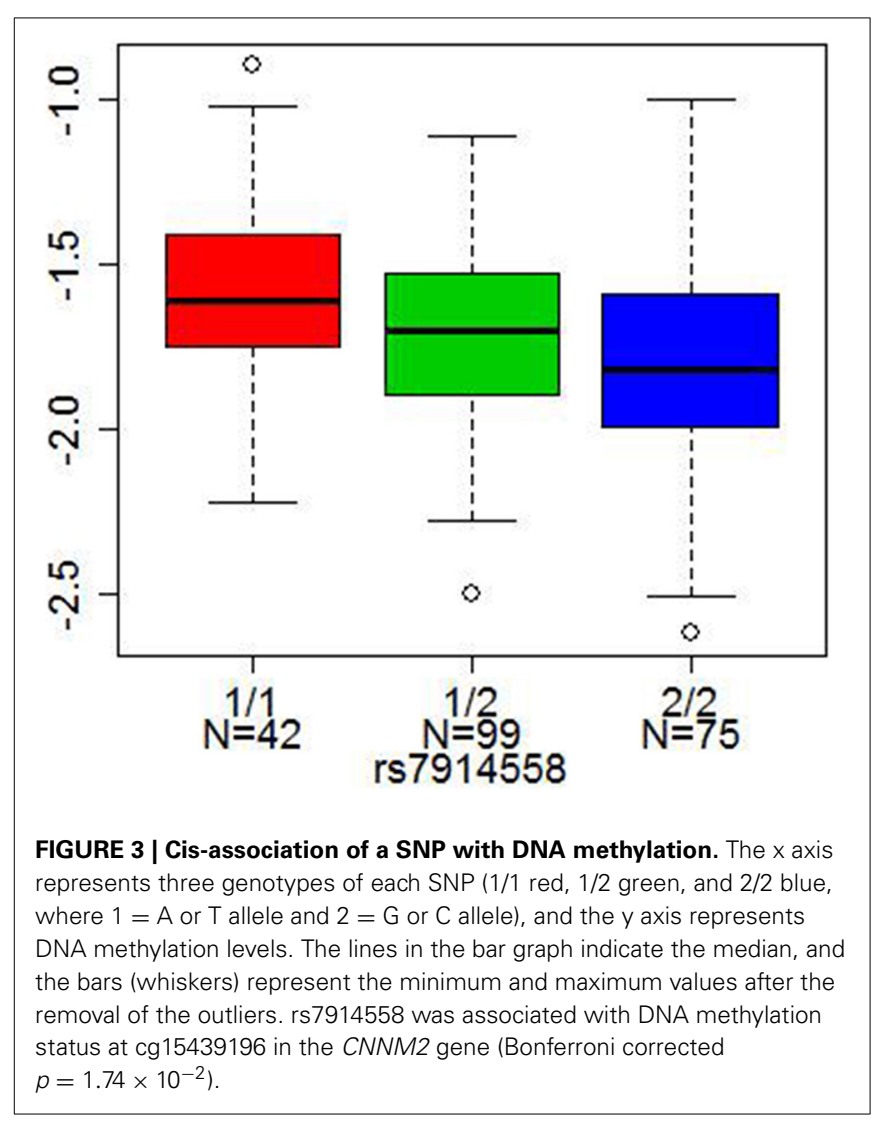

frontal cortex post-mortem brain tissue from 24 patients with schizophrenia and 24 unaffected controls using Illumina Infinium HumanMethylation450 BeadChip (Wockner et al., 2014). Of 4641 probes they identified, 198 common probes were used between Infinium HumanMethylation27 BeadChip and Infinium HumanMethylation450 BeadChip. Of 198 probes, we found one common probe between studies in the FAM5C (family with sequence similarity 5, member C) gene, also known as BRINP3, which showed significantly higher- DNA methylation changes in schizophrenia in both studies. BRINP family is developmentally regulated neural-specific proteins (Kawano et al., 2004), and BRINP3 is related to proliferation and migration (Shorts-Cary et al., 2007).

Finally, this study revealed a large number of cis-mQTLs in the human prefrontal cortex, including risk SNPs associated with schizophrenia in recent genome-wide association studies (Stefansson et al., 2009; Schizophrenia Psychiatric Gnome-Wide Association Study Consortium, 2011). This data set can be useful to reveal potential roles of previously identified risk SNPs associated with schizophrenia as well as discover novel target genes in schizophrenia.

There are several limitations to the present study. First, the coverage of transcriptome by mRNA expression probes and the coverage of methylome by $\mathrm{CpG}$ site probes were limited in this study. Especially, more comprehensive methylome mapping, including $\mathrm{CpG}$ sites outside $\mathrm{CpG}$ islands and intragenic regions will be needed to do a broader analysis of DNA methylome in schizophrenia. We have already initiated such a study. Second, we used genomic DNA extracted from the heterogeneous mixture of various cell types. However, cell type-specific variations in DNA methylation have been documented (Iwamoto et al., 2011) and thus cell type-specific methylome mapping should be performed. Third, we did not investigate histone modifications which are also known to play a role in regulating gene expression (Berger, 2007). Moreover, it is not possible to differentiate methylation from 5-hydroxymethylation of cytosine which also plays a critical role in gene regulation (Bhutani et al., 2011). Finally, it is possible that despite our efforts to remove confounding variables, batch effects and other factors, including smoking and body mass index (Breitling et al., 2011; Dick et al., 2014) might have contributed to the observed differences between the diagnostic groups.

Despite these limitations, our results suggest that altered DNA methylation might be involved in the pathophysiology and/or treatment of schizophrenia, and that a combination of epigenetic and genetic approaches will be useful to understanding the molecular mechanism of this complex disease.

\section{AUTHOR CONTRIBUTIONS}

Shusuke Numata and Barbara K. Lipska designed the experiments and contributed to the interpretation of the results; Shusuke Numata and Barbara K. Lipska performed experiments; Shusuke Numata, Barbara K. Lipska, and Tianzhang Ye analyzed the data; Shusuke Numata, Mary Herman, and Barbara K. Lipska wrote the paper.

\section{ACKNOWLEDGMENTS}

We thank Drs. Llewellyn Bigelow and Joel E. Kleinman for their diagnostic contributions, Dr. Thomas M. Hyde for consenting and dissecting tissues, and Liqin Wang and Vesna Imamovic for their technical expertise. We also thank Dr. Abdel Elkahloun at NHGRI Microarray Core Facility for scanning microarrays. We thank the Offices of the Chief Medical Examiner of Washington, DC and of Northern Virginia-Northern District for their services Dr. Maree Webster from the Stanley Medical Research Institute for their collection of brain samples. We also thank the families of the deceased for their donations of brain tissue and for the time and effort that they devoted to the consent process and interviews. This research was supported by the Intramural Research Program of the National Institute of Mental Health at the National Institutes of Health.

\section{SUPPLEMENTARY MATERIAL}

The Supplementary Material for this article can be found online at: http://www.frontiersin.org/journal/10.3389/fgene. 2014.00280/abstract

\section{REFERENCES}

Abdolmaleky, H. M., Cheng, K. H., Faraone, S. V., Wilcox, M., Glatt, S. J., Gao, F., et al. (2006). Hypomethylation of MB-COMT promoter is a major risk factor for schizophrenia and bipolar disorder. Hum. Mol. Genet. 15, 3132-3145. doi: 10.1093/hmg/ddl253

Abdolmaleky, H. M., Cheng, K. H., Russo, A., Smith, C. L., Faraone, S. V., Wilcox, M., et al. (2005). Hypermethylation of the reelin (RELN) promoter in the brain of schizophrenic patients: a preliminary report. Am. J. Med. Genet. B Neuropsychiatr. Genet. 134B, 60-66. doi: 10.1002/ajmg.b.30140 
Abdolmaleky, H. M., Yaqubi, S., Papageorgis, P., Lambert, A. W., Ozturk, S., Sivaraman, V., et al. (2011). Epigenetic dysregulation of HTR2A in the brain of patients with schizophrenia and bipolar disorder. Schizophr. Res. 129, 183-190. doi: 10.1016/j.schres.2011.04.007

Adkins, D. E., Aberg, K., McClay, J. L., Bukszár, J., Zhao, Z., Jia, P., et al. (2011). Genomewide pharmacogenomic study of metabolic side effects to antipsychotic drugs. Mol. Psychiatry 16, 321-332. doi: 10.1038/mp.2010.14

Allen, N. C., Bagade, S., McQueen, M. B., Ioannidis, J. P. A., Kavvoura, F. K., Khoury, M. J., et al. (2008). Systematic meta-analyses and field synopsis of genetic association studies in schizophrenia: the SzGene database. Nat. Genet. 40, 827-834. doi: 10.1038/ng.171

Anglin, R. E., Mazurek, M. F., Tarnopolsky, M. A., and Rosebush, P. I. (2012). The mitochondrial genome and psychiatric illness. Am. J. Med. Genet. B Neuropsychiatr. Genet. 159B, 749-759. doi: 10.1002/ajmg.b.32086

Berger, S. L. (2007). The complex language of chromatin regulation during transcription. Nature 447, 407-412. doi: 10.1038/nature05915

Bhutani, N., Burns, D. M., and Blau, H. M. (2011). DNA demethylation dynamics. Cell 146, 866-872. doi: 10.1016/j.cell.2011.08.042

Bibikova, M., Le, J., Barnes, B., Saedinia-Melnyk, S., Zhou, L., Shen, R., et al. (2009). Genome-wide DNA methylation profiling using Infinium ${ }^{\circledR}$ assay Epigenomics 1, 177-200. doi: 10.2217/epi.09.14

Bis, J. C., DeCarli, C., Smith, A. V., van der Lijn, F., Crivello, F., Fornage, M., et al. (2012). Common variants at 12 q14 and 12q24 are associated with hippocampal volume. Nat. Genet. 44, 545-549. doi: 10.1038/ng.2237

Breitling, L. P., Yang, R., Korn, B., Burwinkel, B., and Brenner, H. (2011). Tobaccosmoking-related differential DNA methylation: $27 \mathrm{~K}$ discovery and replication. Am. J. Hum. Genet. 88, 450-457. doi: 10.1016/j.ajhg.2011.03.003

Christensen, B. C., Houseman, E. A., Marsit, C. J., Zheng, S., Wrensch, M. R., Wiemels, J. L., et al. (2009). Aging and environmental exposures alter tissuespecific DNA methylation dependent upon CpG island context. PLoS Genet. 5:e1000602. doi: 10.1371/journal.pgen.1000602

Cohen, S. M., Nichols, A., Wyatt, R., and Pollin, W. (1974). The administration of methionine to chronic schizophrenic patients: a review of ten studies. Biol. Psychiatry 8, 209-225.

Davies, M. N., Volta, M., Pidsley, R., Lunnon, K., Dixit, A., Lovestone, S., et al. (2012). Functional annotation of the human brain methylome identifies tissuespecific epigenetic variation across brain and blood. Genome Biol. 13:R43. doi: 10.1186/gb-2012-13-6-r43

Dempster, E. L., Mill, J., Craig, I. W., and Collier, D. A. (2006). The quantification of COMT mRNA in post mortem cerebellum tissue: diagnosis, genotype, methylation and expression. BMC Med. Genet. 7:10. doi: 10.1186/14712350-7-10

Dempster, E. L., Pidsley, R., Schalkwyk, L. C., Owens, S., Georgiades, A., Kane, F., et al. (2011). Disease-associated epigenetic changes in monozygotic twins discordant for schizophrenia and bipolar disorder. Hum. Mol. Genet. 20, 4786-4796. doi: 10.1093/hmg/ddr416

Dick, K. J., Nelson, C. P., Tsaprouni, L., Sandling, J. K., Aïssi, D., Wahl, S., et al. (2014). DNA methylation and body-mass index: a genome-wide analysis. Lancet 383, 1990-1998. doi: 10.1016/S0140-6736(13)62674-4

Fijal, B. A., Stauffer, V. L., Kinon, B. J., Conley, R. R., Hoffmann, V. P., Witte, M. M., et al. (2012). Analysis of gene variants previously associated with iloperidone response in patients with schizophrenia who are treated with risperidone. J. Clin. Psychiatry 73, 367-371. doi: 10.4088/JCP.10m06507

Gamazon, E. R., Badner, J. A., Cheng, L., Zhang, C., Zhang, D., Cox, N. J., et al. (2013). Enrichment of cis-regulatory gene expression SNPs and methylation quantitative trait loci among bipolar disorder susceptibility variants. Mol. Psychiatry 18, 340-346. doi: 10.1038/mp.2011.174

Gibbs, J. R., van der Brug, M. P., Hernandez, D. G., Traynor, B. J., Nalls, M. A., Lai, S. L., et al. (2010). Abundant quantitative trait Loci exist for DNA methylation and gene expression in human brain. PLoS Genet. 6:e1000952. doi: 10.1371/journal.pgen.1000952

Glessner, J. T., Wang, K., Cai, G., Korvatska, O., Kim, C. E., Wood, S., et al. (2009). Autism genome-wide copy number variation reveals ubiquitin and neuronal genes. Nature 459, 569-573. doi: 10.1038/nature07953

Grayson, D. R., Jia, X., Chen, Y., Sharma, R. P., Mitchell, C. P., Guidotti, A., et al. (2005). Reelin promoter hypermethylation in schizophrenia. Proc. Natl. Acad. Sci. U.S.A. 102, 9341-9846. doi: 10.1073/pnas.0503736102

Irizarry, R. A., Ladd-Acosta, C., Wen, B., Wu, Z., Montano, C., Onyango, P., et al. (2009). The human colon cancer methylome shows similar hypo- and hypermethylation at conserved tissue-specific CpG island shores. Nat. Genet. 41, 178-186. doi: 10.1038/ng.298

Iwamoto, K., Bundo, M., Ueda, J., Oldham, M. C., Ukai, W., Hashimoto, E., et al. (2011). Neurons show distinctive DNA methylation profile and higher interindividual variations compared with non-neurons. Genome Res. 21, 688-696. doi: 10.1101/gr.112755.110

Iwamoto, K., Bundo, M., Yamada, K., Takao, H., Iwayama-Shigeno, Y., Yoshikawa, T., et al. (2005). DNA methylation status of SOX10 correlates with its downregulation and oligodendrocyte dysfunction in schizophrenia. J. Neurosci. 25, 5376-5381. doi: 10.1523/JNEUROSCI.0766-05.2005

Jamadar, S., Powers, N. R., Meda, S. A., Calhoun, V. D., Gelernter, J., Gruen, J. R., et al. (2013). Genetic influences of resting state fMRI activity in language-related brain regions in healthy controls and schizophrenia patients: a pilot study. Brain Imaging Behav. 7, 15-27. doi: 10.1007/s11682-012-9168-1

Jamadar, S., Powers, N. R., Meda, S. A., Gelernter, J., Gruen, J. R., and Pearlson, G. D. (2011). Genetic influences of cortical gray matter in language-related regions in healthy controls and schizophrenia. Schizophr. Res. 129, 141-148. doi: 10.1016/j.schres.2011.03.027

Kawano, H., Nakatani, T., Mori, T., Ueno, S., Fukaya, M., Abe, A., et al. (2004). Identification and characterization of novel developmentally regulated neuralspecific proteins, BRINP family. Brain Res. Mol. Brain Res. 125, 60-75. doi: 10.1016/j.molbrainres.2004.04.001

Kinoshita, M., Numata, S., Tajima, A., Shimodera, S., Ono, S., Imamura, A., et al. (2013). DNA methylation signatures of peripheral leukocytes in schizophrenia. Neuromolecular Med. 15, 95-101. doi: 10.1007/s12017-0128198-6

Lavedan, C., Licamele, L., Volpi, S., Hamilton, J., Heaton, C., Mack, K., et al. (2009). Association of the NPAS3 gene and five other loci with response to the antipsychotic iloperidone identified in a whole genome association study. Mol. Psychiatry 14, 804-819. doi: 10.1038/mp.2008.56

Leek, J. T., and Storey, J. D. (2007). Capturing heterogeneity in gene expression studies by surrogate variable analysis. PLoS Genet. 3, 1724-1735. doi: 10.1371/journal.pgen.0030161

Lesch, K. P., Timmesfeld, N., Renner, T. J., Halperin, R., Röser, C., Nguyen, T. T., et al. (2008). Molecular genetics of adult ADHD: converging evidence from genome-wide association and extended pedigree linkage studies. J. Neural Transm. 115, 1573-1585. doi: 10.1007/s00702-008-0119-3

Lionel, A. C., Crosbie, J., Barbosa, N., Goodale, T., Thiruvahindrapuram, B., Rickaby, J., et al. (2011). Rare copy number variation discovery and cross-disorder comparisons identify risk genes for ADHD. Sci. Transl. Med. 3, 95ra75. doi: 10.1126/scitranslmed.3002464

Lipska, B. K., Deep-Soboslay, A., Weickert, C. S., Hyde, T. M., Martin, C. E., Herman, M. M., et al. (2006). Critical factors in gene expression in postmortem human brain: focus on studies in schizophrenia. Biol. Psychiatry 60, 650-658. doi: 10.1016/j.biopsych.2006.06.019

Liu, C., Cheng, L., Badner, J. A., Zhang, D., Craig, D. W., Redman, M., et al. (2010). Whole-genome association mapping of gene expression in the human prefrontal cortex. Mol. Psychiatry 15, 779-784. doi: 10.1038/mp.2009.128

Makino, C., Fujii, Y., Kikuta, R., Hirata, N., Tani, A., Shibata, A., et al. (2003). Positive association of the AMPA receptor subunit GluR4 gene (GRIA4) haplotype with schizophrenia: linkage disequilibrium mapping using SNPs evenly distributed across the gene region. Am. J. Med. Genet. B Neuropsychiatr. Genet. 116B, 17-22. doi: 10.1002/ajmg.b.10041

Meng, H., Smith, S. D., Hager, K., Held, M., Liu, J., Olson, R. K., et al. (2005). DCDC2 is associated with reading disability and modulates neuronal development in the brain. Proc. Natl. Acad. Sci. U.S.A. 102, 17053-17058. doi: 10.1073/pnas.0508591102

Mill, J., Tang, T., Kaminsky, Z., Khare, T., Yazdanpanah, S., Bouchard, L., et al. (2008). Epigenomic profiling reveals DNA-methylation changes associated with major psychosis. Am. J. Hum. Genet. 82, 696-711. doi: 10.1016/j.ajhg.2008.01.008

Numata, S., Ye, T., Thomas, T. M., Guitart-Navarro, X., Tao, R., Wininger, M., et al. (2012). Epigenetic modifications in development and aging of the human prefrontal cortex. Am. J. Hum. Genet. 90, 260-272. doi: 10.1016/j.ajhg. 2011.12.020

Petronis, A., Gottesman, II., Kan, P., Kennedy, J. L., Basile, V. S., Paterson, A. D., et al. (2003). Monozygotic twins exhibit numerous epigenetic differences: clues to twin discordance? Schizophr. Bull. 29, 169-178. doi: 10.1093/oxfordjournals.schbul.a006988 
Pollin, W., Cardon, P. V. Jr., and Kety, S. S. (1961). Effects of amino acid feedings in schizophrenic patients treated with iproniazid. Science 133, 104-105. doi: 10.1126/science.133.3446.104

Purcell, S., Neale, B., Todd-Brown, K., Thomas, L., Ferreira, M. A., Bender, D., et al. (2007). PLINK: a tool set for whole-genome association and population-based linkage analyses. Am. J. Hum. Genet. 81, 559-575. doi: 10.1086/519795

Ruzicka, W. B., Zhubi, A., Veldic, M., Grayson, D. R., Costa, E., and Guidotti, A. (2007). Selective epigenetic alteration of layer I GABAergic neurons isolated from prefrontal cortex of schizophrenia patients using laser-assisted microdissection. Mol. Psychiatry 12, 385-397. doi: 10.1038/sj.mp.4001954

Sagata, N., Iwaki, A., Aramaki, T., Takao, K., Kura, S., Tsuzuki, T., et al. (2010). Comprehensive behavioural study of GluR4 knockout mice: implication in cognitive function. Genes Brain Behav. 9, 899-909. doi: 10.1111/j.1601183X.2010.00629.x

Schizophrenia Psychiatric Gnome-Wide Association Study Consortium. (2011). Genome-wide association study identifies five new schizophrenia loci. Nat. Genet. 43, 969-976. doi: 10.1038/ng.940

Shi, J., Levinson, D. F., Duan, J., Sanders, A. R., Zheng, Y., Pe'er, I., et al. (2009). Common variants on chromosome $6 \mathrm{p} 22.1$ are associated with schizophrenia. Nature 460, 753-757. doi: 10.1038/nature08192

Shorts-Cary, L., Xu, M., Ertel, J., Kleinschmidt-Demasters, B. K., Lillehei, K., Matsuoka, I., et al. (2007). Bone morphogenetic protein and retinoic acidinducible neural specific protein-3 is expressed in gonadotrope cell pituitary adenomas and induces proliferation, migration, and invasion. Endocrinology 148, 967-975. doi: 10.1210/en.2006-0905

Smith, R. M., Papp, A. C., Webb, A., Ruble, C. L., Munsie, L. M., Nisenbaum, L. K., et al. (2013). Multiple regulatory variants modulate expression of 5Hydroxytryptamine 2A receptors in human cortex. Biol. Psychiatry 73, 546-554. doi: 10.1016/j.biopsych.2012.09.028

Stefansson, H., Ophoff, R. A., Steinberg, S., Andreassen, O. A., Cichon, S., Rujescu, D., et al. (2009). Common variants conferring risk of schizophrenia. Nature 460, 744-747. doi: 10.1038/nature08186

Tamura, Y., Kunugi, H., Ohashi, J., and Hohjoh, H. (2007). Epigenetic aberration of the human REELIN gene in psychiatric disorders. Mol. Psychiatry 12, 519, 593-600. doi: 10.1038/sj.mp.4001965

Tochigi, M., Iwamoto, K., Bundo, M., Komori, A., Sasaki, T., Kato, N., et al. (2008). Methylation status of the reelin promoter region in the brain of schizophrenic patients. Biol. Psychiatry 63, 530-533. doi: 10.1016/j.biopsych.2007. 07.003

Tolosa, A., Sanjuán, J., Dagnall, A. M., Moltó, M. D., Herrero, N., and de Frutos, R. (2010). FOXP2 gene and language impairment in schizophrenia: association and epigenetic studies. BMC Med. Genet. 11:114. doi: 10.1186/1471-235011-114

Tremolizzo, L., Carboni, G., Ruzicka, W. B., Mitchell, C. P., Sugaya, I., Tueting, P., et al. (2002). An epigenetic mouse model for molecular and behavioral neuropathologies related to schizophrenia vulnerability. Proc. Natl. Acad. Sci. U.S.A. 99, 17095-17100. doi: 10.1073/pnas.262658999

Tremolizzo, L., Doueiri, M. S., Dong, E., Grayson, D. R., Davis, J., Pinna, G., et al. (2005). Valproate corrects the schizophrenia-like epigenetic behavioral modifications induced by methionine in mice. Biol. Psychiatry 57, 500-509. doi: 10.1016/j.biopsych.2004.11.046

Veldic, M., Caruncho, H. J., Liu, W. S., Davis, J., Satta, R., Grayson, D. R., et al. (2004). DNA-methyltransferase $1 \mathrm{mRNA}$ is selectively overexpressed in telencephalic GABAergic interneurons of schizophrenia brains. Proc. Natl. Acad. Sci. U.S.A. 101, 348-353. doi: 10.1073/pnas.2637013100

Veldic, M., Guidotti, A., Maloku, E., Davis, J. M., and Costa, E. (2005). In psychosis, cortical interneurons overexpress DNA-methyltransferase 1. Proc. Natl. Acad. Sci. U.S.A. 102, 2152-2157. doi: 10.1073/pnas.0409665102

Vrijenhoek, T., Buizer-Voskamp, J. E., van der Stelt, I., Strengman, E., Genetic Risk and Outcome in Psychosis (GROUP) Consortium, Sabatti, C., et al. (2008) Recurrent CNVs disrupt three candidate genes in schizophrenia patients. Am. J. Hum. Genet. 83, 504-510. doi: 10.1016/j.ajhg.2008.09.011

Wang, K. S., Liu, X. F., and Aragam, N. (2010). A genome-wide meta-analysis identifies novel loci associated with schizophrenia and bipolar disorder. Schizophr. Res. 124, 192-199. doi: 10.1016/j.schres.2010.09.002

Wilson, P. M., Fryer, R. H., Fang, Y., and Hatten, M. E. (2010). Astn2, a novel member of the astrotactin gene family, regulates the trafficking of ASTN1 during glial-guided neuronal migration. J. Neurosci. 30, 8529-8540. doi: 10.1523/JNEUROSCI.0032-10.2010

Wockner, L. F., Noble, E. P., Lawford, B. R., Young, R. M., Morris, C. P., Whitehall, V. L., et al. (2014). Genome-wide DNA methylation analysis of human brain tissue from schizophrenia patients. Transl. Psychiatry 4, e339. doi: 10.1038/tp.2013.111

Yang, C. P., Wang, H. A., Tsai, T. H., Fan, A., Hsu, C. L., Chen, C. J., et al. (2012). Characterization of the neuropsychological phenotype of glycine $\mathrm{N}$-methyltransferase-/- mice and evaluation of its responses to clozapine and sarcosine treatments. Eur. Neuropsychopharmacol. 22, 596-606. doi: 10.1016/j.euroneuro.2011.12.007

Ye, T., Lipska, B. K., Tao, R., Hyde, T. M., Wang, L., Li, C., et al. (2012). Analysis of copy number variations in brain DNA from patients with schizophrenia and other psychiatric disorders. Biol. Psychiatry 72, 651-654. doi: 10.1016/j.biopsych.2012.06.014

Zhang, D., Cheng, L., Badner, J. A., Chen, C., Chen, Q., Luo, W., et al. (2010). Genetic control of individual differences in gene-specific methylation in human brain. Am. J. Hum. Genet. 86, 411-419. doi: 10.1016/j.ajhg.2010.02.005

Zhubi, A., Veldic, M., Puri, N. V., Kadriu, B., Caruncho, H., Loza, I., et al. (2009). An upregulation of DNA-methyltransferase 1 and 3a expressed in telencephalic GABAergic neurons of schizophrenia patients is also detected in peripheral blood lymphocytes. Schizophr. Res. 111, 115-122. doi: 10.1016/j.schres.2009.03.020

Conflict of Interest Statement: The authors declare that the research was conducted in the absence of any commercial or financial relationships that could be construed as a potential conflict of interest.

Received: 09 June 2014; accepted: 31 July 2014; published online: 26 August 2014. Citation: Numata S, Ye T, Herman M and Lipska BK (2014) DNA methylation changes in the postmortem dorsolateral prefrontal cortex of patients with schizophrenia. Front. Genet. 5:280. doi: 10.3389/fgene.2014.00280

This article was submitted to Neurogenomics, a section of the journal Frontiers in Genetics.

Copyright (c) 2014 Numata, Ye, Herman and Lipska. This is an open-access article distributed under the terms of the Creative Commons Attribution License (CC BY). The use, distribution or reproduction in other forums is permitted, provided the original author(s) or licensor are credited and that the original publication in this journal is cited, in accordance with accepted academic practice. No use, distribution or reproduction is permitted which does not comply with these terms. 